Sharif University of Technology
Scientia Iranica
Transactions E: Industrial Engineering
hCIENTIA

\title{
Synchronized timetables for bus rapid transit networks in small and large cities
}

\author{
Sh. Ataeian ${ }^{\mathrm{a}}$, M. Solimanpur ${ }^{\mathrm{a}, *}$, S.M.M. Amiripour ${ }^{\mathrm{b}}$, and R. Shankar \\ a. Department of Industrial Engineering, Urmia University, Urmia, Iran. \\ b. Toos Institute of Higher Education, Mashhad, Iran. \\ c. Department of Management Studies, Indian Institute of Technology Delhi, New Delhi, India.
}

Received 10 August 2018; received in revised form 21 February 2019; accepted 20 July 2019

\section{KEYWORDS}

Public transportation; Bus line timetable setting;

Mathematical modeling;

Mixed-integer programming; Genetic algorithm.

\begin{abstract}
The quality of public transportation service has significant effects on the quality of urban life. In the course of frequency setting and timetabling as an important step in the public transportation planning process, synchronization gains significance and directly influences the utility and attractiveness of the system; therefore, a great deal of attention should be drawn to it in the whole planning process, especially in setting frequency and timetable. To this end, the present study proposes a mixed-integer nonlinear programming model to set timetables on a bus transit network with maximum synchronization and minimum number of fleet size. The proposed model is applicable to both small- and large-scale transit networks and is used for setting timetables on two samples of different sizes. A simple problem in this study was solved by General Algebraic Modeling System (GAMS) Software where the obtained timetable seemed quite reasonable. Moreover, the proposed model was employed to set timetables through the genetic algorithm on Tehran Bus Rapid Transit (BRT) networks as a real-life instance; then, the NSGA-II was used to obtain the Pareto optimal solutions of the problem for five different scenarios. Finally, the results showed that the proposed model was efficient in setting timetables on transit networks of different sizes.
\end{abstract}

(C) 2021 Sharif University of Technology. All rights reserved.

\section{Introduction}

Public transportation is fundamental to every city. The quality of the public transportation system in a city directly affects the quality of urban life. While a reliable public transportation system can attract a considerable number of daily passengers, a poor public transit service can considerably reduce them [1]. Nowadays, daily commute of a significant number of

*. Corresponding author.

E-mail addresses: st_sh.ataieyan@urmia.ac.ir (Sh.

Ataeian); m.solimanpur@urmia.ac.ir (M. Solimanpur);

amiripour@toos.ac.ir (S.M.M. Amiripour);

ravi1@dms.iitd.ac.in (R. Shankar) people, especially those with low income, is directly dependent on the public transportation infrastructures. Among different modes of public transportation, buses are the most common ones due to their flexibility, compatibility, and required investment. Therefore, followed by urban expansion and emergence of new modes of transportation thanks to the growing technology, further studies should be conducted to improve urban transportation, especially bus systems.

The process of urban bus planning is quite complicated and requires making interdependent decisions. According to [2-8], this process can be divided into four basic steps:

1. Designing network routes;

2. Frequency setting and timetabling; 
3. Scheduling the vehicles;

4. Assigning the crew.

Each step is a complicated process and consists of several operations; therefore, they are usually performed in sequence, i.e., the output of one step will be the input for the next one. The present paper focuses on the second step of the urban bus planning process, i.e., frequency setting and timetabling.

Frequency setting deals with determining an efficient service frequency for each line based on the time of day and day of the week. In timetable setting, the scheduler prepares a list of departure times according to the corresponding headways of each line and estimates the arrival time of each departure for each stop in each line. The scheduler should also consider available resources such as available fleet size. Timetables provide information about the departure and arrival times of services on each line for passengers. Having this information, passengers can make better decisions on arriving services at the stations and adapt themselves to the bus departure times. In other words, while having a timetable at hand, passengers know when they would arrive at a stop and minimize their waiting time. Setting urban bus timetable may arise several problems that have received much attention because of its significance in the literature.

The quality of urban bus system depends considerably on the service timekeeping and arrival times of vehicles. However, arrival times are easily affected by the number of passengers who use the system, and the bus schedule is closely related to the dynamic motion of buses with passengers $[9,10]$. To present an efficient service, planners are responsible for synchronization of the schedules of different lines in a transit network. Proper synchronization can be achieved by maximizing the number of simultaneous arrivals of different departures of different lines at transfer points. Petersen et al. [11] suggested that the average waiting time for a transfer be about 9.75 minutes on weekends in the Greater Copenhagen area. Synchronization decreases the waiting times of passengers transferring among lines of a network, thus leading to a higher level of service and encouraging more people to use public transportation. Therefore, synchronization is a common objective that has gained much attention and has been studied in other modes of transportation such as urban railway networks $[12,13]$.

Ceder et al. [14] proposed an optimization model with the objective of maximizing the number of simultaneous arrivals in which the problem was formulated as a mixed-integer programming problem, and a heuristic algorithm was developed to solve it. They defined simultaneous arrivals as the arrival of two departures from different lines to a transfer point at the same time. Eranki [15] extended the model developed by Ceder et al. [14] by adding a time window to the simultaneity definition and proposed a model for timetable setting with the same objective. The new model was also formulated as a mixed-integer linear programming problem solved by a heuristic algorithm. The model proposed by Ceder et al. [14] has received much attention and has been extended further by other researchers [16-18]. In this regard, a number of studies have been devoted to minimizing the transfer time [19-24]. Gao et al. [25] proposed a bi-level programming technique to deal with the problem of frequency setting. The upper-level problem considers minimizing an objective that consists of in-vehicle and waiting time and frequency setting cost. The lowerlevel problem took into account the alternative paths for passengers. A heuristic algorithm based on the sensitivity analysis was also developed to solve the model and set optimal frequencies.

Bookbinder and Désilets [26] proposed a transfer optimization model in which travel times were regarded stochastic and followed a truncated exponential distribution. They aimed to minimize the total passenger inconvenience. Furthermore, a heuristic algorithm was developed by Ting and Schonfeld [27] considering slack times to optimize the connecting lines. Knoppers and Muller [28] studied the chances of optimized transfer in order to minimize passenger transfer times. Considering the probabilities and limitations of the synchronized transfers, they suggested that optimal synchronization could be hard to achieve in case of high frequencies at crossing lines. The synchronization strategy proposed in their study enables the vehicle to handle the second part of the trip to delay its departure if the transfer passengers are about to arrive soon; therefore, transfer times are often increased. Yu et al. [29] proposed an optimization model for bus transit networks with the objective of minimizing the transfer and maximizing the passenger flow per unit length. A Coarse-grain Parallel Ant Colony Algorithm (CPACA) was employed to solve the problem. The results showed that achieving a bus transit network with optimal transfers and shorter waiting times would not be farfetched. Ant colony algorithm is frequently used by other researchers as well [30,31]. Heuristic approaches have also been used by a number of researchers for transfer optimization and synchronization among other steps of the urban transportation process [32]. Fleurent et al. [33] used the idea of weighted transfers to describe the concepts used in Hastus commercial software for producing synchronized timetables. Furthermore, concentrating on minimizing the transfer waiting times, Castelli et al. [34] proposed a mathematical model for scheduling problems. As one of the features of the proposed model, the number of dispatches does not depend on the frequency bounds, but on the service quality and its costs. Service quality is evaluated by the 
sum of the transfer waiting times of all the passengers whose boarding and alighting times should be available. However, the authors approve that despite the limited suppositions, their model cannot be used for optimizing a large-scale network because of its numerous variables; therefore, a Lagrangian heuristic method was also proposed. Rapp and Gehner [35] proposed a heuristic method for optimizing the transfer delays. The survey describes four coordinated processes for practical transit planning. The operational tool used for optimizing the transfer delays changes the departure times from the terminal automatically and iteratively. The results indicated that in comparison to manual scheduling, the optimized timetable could efficiently minimize the total transfer delay times and cause no additional cost. Chakroborty et al. [22,23] focused on the application of Genetic Algorithms (GA) in determining the waiting time in transit networks in order to minimize the total passenger waiting times. The proposed mixed-integer nonlinear model was formulated and a hybrid GA was selected for implementation purposes. The genetic display of a thorough timetable consists of a set of binary numbers that represent the headways and stop times of the successive vehicles on all lines. However, as already mentioned, the proposed display might not be suitable for large transit networks.

The GA has also been applied to bus scheduling problems. Deb and Chakroborty [36] formulated an optimization problem for a bus transit system with the objective of minimizing the total passenger waiting times (with or without transfers) while satisfying the resource and service constraints. According to the conducted studies, the GA is ideal for such problems. Chakroborty et al. [37] combined transfer synchronization with vehicle scheduling as a mixedinteger nonlinear problem. The problem, aimed at determining the fleet size and setting timetables to minimize the passenger waiting times, was solved using the GA. Of note, the problem was limited to one transfer point, i.e., the intersection of multiple lines, and the genetic display seemed to be not suitable for large problems. Ngamchai and Lovell [38] presented a new transfer optimization model for designing the bus transit route and solved it using the GA. The bus routes were designed in two phases. The route improvement algorithm employed genetic operators and in conjunction, another heuristic approach was used for headway synchronization in order to improve the system efficiency. The route improvement algorithm reduces both transfer times and total cost. Moreover, headway synchronization methods are generally used for service frequency setting. Cevallos and Zhao [20] proposed an approach based on GA at the network level for bus transfer time optimization. The main objective of the proposed algorithm was to find the best possible solution to the problem of transfer time optimization through timetable movements. They aimed to minimize the transfer waiting times of an existing timetable under strict evenly spaced headway constraints. The results indicated that the proposed algorithm could efficiently shorten the waiting times. In conjunction with these studies, GA are widely used for different aspects of transit planning and optimization [39-47]. Table 1 presents a summary of the aforementioned research papers.

As stated earlier, the mathematical model developed by Ceder et al. [14] has been used as a precedent by many researchers. The present study develops a model to increase the simultaneous arrivals of buses in the network as first developed by Ceder et al. [14]. Moreover, a different definition of simultaneous arrivals was suggested which is more practical, effective, and realistic. The objective function of maximizing the simultaneous arrivals and considering a time window as an allowable deadline for simultaneity are the concepts that have been borrowed from previous studies [14,15]. The problem of timetable setting is formulated as a mathematical programming model whose implementation and solution methods on both small- and largescale networks are separately discussed and exemplified.

The rest of this paper is organized as follows. Section 2 presents a definition of the timetable setting problem with optimal synchronization. Section 3 discusses the procedure of formulating the problem as a mathematical programming model. Section 4.1 briefly elaborates on the scale of the problem. Section 4.2 shows how to convert the proposed bi-objective problem into a single objective one. Sections 4.3 and 4.4 introduce the numerical examples of both small- and large-scale networks, respectively. Finally, Section 5 discusses the results and concludes the paper.

\section{Problem definition}

Different aspects of timetable setting problem make one consider different objectives for the problem from different angles. One of these significant objectives, according to Ceder et al. [14], is to minimize the transfer waiting times that can be achieved by maximizing the number of simultaneous arrivals. Since all of the origins and destinations are not directly connected in a large public transit network, several transfer points are required to cover the utmost area. However, an extra waiting time is imposed on the passengers who intend to travel among different lines of a network via transfer points. A perfect timetable is set only when the waiting time at transfer points is minimized by the simultaneous arrival of vehicles at transfer points. Figure 1 illustrates the significance of transfer points and simultaneous arrivals at transfer points. As shown in Figure 1, a passenger who intends to travel from 
Table 1. Literature summary.

\begin{tabular}{|c|c|c|c|c|}
\hline Ref. & Year & Author(s) & Objective & Solution approach \\
\hline$[14]$ & 2001 & Ceder et al. & Number of simultaneous arrivals & Heuristic approach \\
\hline$[15]$ & 2004 & Eranki & $\begin{array}{l}\text { Number of simultaneous arrivals, } \\
\text { passenger waiting time }\end{array}$ & Heuristic approach \\
\hline$[17]$ & 2003 & Quak & $\begin{array}{l}\text { Drive time, number of line runs, } \\
\text { detour time, overloading, } \\
\text { fleet size, waiting time }\end{array}$ & Heuristic approach \\
\hline$[20]$ & 2006 & Cevallos and Zhao & Transfer waiting time & Evolutionary \\
\hline$[21]$ & 2002 & Jansen et al. & Passenger transfer times & Neighborhood search \\
\hline$[22,23,36]$ & $1995 / 1997 / 1998$ & Chakroborty et al. & Passengers total waiting time & Evolutionary \\
\hline$[24]$ & 1995 & Daduna and VoB & Transfer time & Neighborhood search \\
\hline$[25]$ & 2004 & Gao et al. & Deterrence, cost, system performance & Heuristic approach \\
\hline$[26]$ & 1992 & Bookbinder and Désilets & Waiting time & - \\
\hline$[29]$ & 2005 & Yu et al. & $\begin{array}{l}\text { Number of transfers, maximum } \\
\text { passenger flow per unit length }\end{array}$ & - \\
\hline [33] & 2004 & Fleurent et al. & $\begin{array}{l}\text { Trip synchronization, historical runs, } \\
\text { fleet size, deadhead, layover }\end{array}$ & Heuristic approach \\
\hline$[34]$ & 2004 & Castelli et al. & Passenger transfer waiting time & Heuristic approach \\
\hline$[35]$ & 1976 & Rapp and Gehner & Transfer optimization & Heuristic approach \\
\hline$[37]$ & 2001 & Chakroborty et al. & $\begin{array}{l}\text { Transfer coordination (waiting time), } \\
\text { fleet size }\end{array}$ & Evolutionary \\
\hline [38] & 2003 & Ngamchai and Lovell & Fleet size, in-vehicle and waiting time & Evolutionary \\
\hline
\end{tabular}

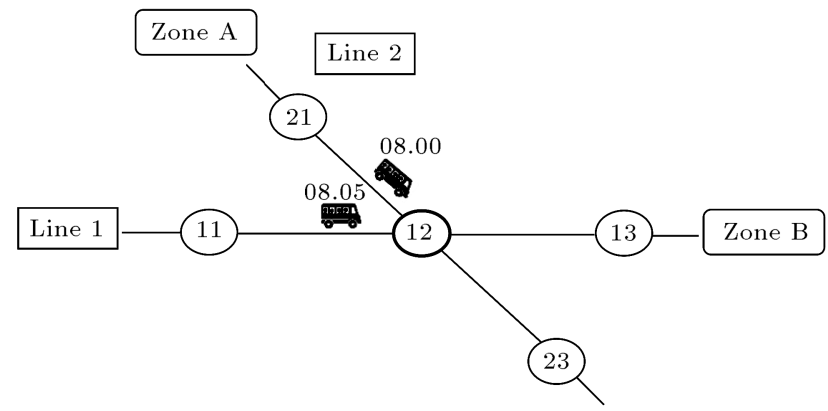

Figure 1. The simultaneous arrival of two departures.

zone A to zone B should be transferred from line 2 to line 1 , since there is no direct link between these two zones. The transfer should be made at stop 12 that is the transfer stop of two lines. If the arrivals of the bus on which the passenger is riding and the bus the passenger needs to catch to continue the trip are not much apart, the transfer waiting time would be short; thus, the trip would remain attractive and convenient for the passenger. However, the arrival times of the buses on each line should also be taken into account. For instance, in this case, when the transfer passenger arrives at stop 12 at 8:00, a short time is required to get off the first bus, walk to the next stop point, and get on the immediate next bus on line 1 which arrives at stop 12 at 8:05. Therefore, the passenger has a short time to move between the buses on the lines. This interval between the buses arriving at the transfer point from different lines should be adjusted according to the time of day and frequency system. Due to the high frequency of the services over peak hours, the time intervals should be quite short to prevent possible delays. However, due to the low frequency of the services during non-peak hours, a slight increase in the waiting time would not considerably affect the trip attractiveness. Furthermore, the interval also depends highly on the overall characteristics of the intersecting lines. For example, it should be kept in mind that if one of the lines is in suburban areas, the service frequency will be generally low on that line even during the peak hours; thus, the interval should not be too short as 
it is for intersecting Central Business District (CBD) lines. In real life, the timetable scheduler cannot expect passengers to plan their trips so as to minimize their transfer time. The only thing they can do is to adapt themselves to the departure times at their origin. In this regard, it is the scheduler's task to synchronize the arrivals at transfer points in order to reduce the transfer time. Besides, the scheduler should plan the departures according to passengers' demands in the process of setting timetables; otherwise, demands of passengers will not be satisfied and vehicles will be delayed while facing longer dwell times, hence bunching phenomenon [2].

A good timetable should be cost-effective; in other words, in addition to satisfying passengers' demands, it should take into account the benefits of the transit company. Resource constraint is an important issue for all transit companies. However, they can attract more passengers by optimizing their current services. If the proposed synchronization objective is considered alone, the optimal solution will set the headways of all lines at their minimum to maximize the service level which will also increase the number of required vehicles. Therefore, another important objective is to minimize the required vehicles. The fleet size is a significant factor that can greatly affect the costs for companies. Headways, as the time between two successive departures, can be evenly or unevenly spaced. If they are evenly spaced, the vehicles depart at regular and equal intervals. In a period that headways are evenly spaced, the number of the required vehicles on a line can be calculated using Eq. (1):

$$
\text { fleet size }=\frac{\text { average round trip time }}{\text { head way }} \text {. }
$$

Considering the above-mentioned objectives, the present study proposes a bi-objective mixed-integer programming model whose mathematical formulation is presented in the next section.

\section{Problem formulation}

The notations used in the mathematical model are presented in Table 2.

The objectives of the problem are formulated as shown in Eqs. (2) and (3). The first objective maximizes the number of synchronized arrivals at transfer points. The second objective minimizes the number of required vehicles for the system.

$$
\max Z_{1}=\sum_{m \in D_{t}^{l}} \sum_{n \in D_{t}^{k}} \sum_{q \in C^{l}} \sum_{l \in L} \sum_{\substack{k \in L \\ l \neq k}} \sum_{t \in T} \sum_{p \in T} y_{m n q t p}^{l k}
$$

$\min Z_{2}=\sum_{l \in L} F S^{l}$

$$
\begin{aligned}
& x_{t}^{l} \leq H \max _{t}^{l} \quad l \in L, t \in T, \\
& x_{t}^{l}+\left(N D_{t}^{l}-1\right) H_{t}^{l} \leq U \quad l \in L, t \in T, \\
& H \min _{t}^{l} \leq H_{t}^{l} \leq H \max _{t}^{l} \quad l \in L, t \in T, \\
& A_{n i t}^{l}=U(t-1)+x_{t}^{l}+(n-1) H_{t}^{l} \\
& +\sum_{k=1, k \in S^{l}}^{i-1} R_{k, k+1, t}^{l}+\sum_{k=1, k \in S^{l}}^{i-1} d w_{k, t}^{l} \\
& l \in L, n \in D_{t}^{l}, i \in S^{l}, t \in T \text {, } \\
& y_{m n q t p}^{l k}= \begin{cases}1 & \left|A_{m q t}^{l}-A_{n q p}^{k}\right| \leq \delta^{l k} \\
0 & \text { otherwise }\end{cases} \\
& l, k \in L, m \in D_{t}^{l}, n \in D_{t}^{k}, q \in C^{l k}, t, p \in T, \\
& l \neq k, l<k \\
& \left.F S^{l}=\max _{t \in T}\left(C T_{t}^{l}\right) / H_{t}^{l}\right) \quad l \in L, \\
& y_{m n q t p}^{l k} \in\{0,1\} \quad l, k \in L, m \in D_{t}^{l}, \quad n \in D_{t}^{k} \text {, } \\
& q \in C^{l k}, t, p \in T, l \neq k, l<k \\
& x_{t}^{l}, H_{t}^{l} \in N^{+}, l \in L, t \in T .
\end{aligned}
$$

The constraints of the model are formulated as Eqs. (4) to (11). Constraint (4) guarantees that the first departure would take place in a time period shorter than the maximum allowable headway. Constraint (5) ensures that the last departure in each period is set before the end of the corresponding period. Constraint (6) limits the headway of each period in order not to exceed the allowable range. Eq. (7) calculates the arrival time of each departed vehicle at each stop. Constraint (8) defines the binary variable y introduced in Table 2 . It compares all the arrival times calculated by Constraint (7). In case the interval between two arrivals from different lines at a transfer point is shorter than the maximum allowable time window $\left(\delta_{t p}^{l k}\right), y$ takes 1 ; otherwise, it remains equal to 0 . Eq. (9) calculates the minimum number of the required vehicles on each line which is equal to the maximum number of the required vehicles in each period on the same line. Constraints (10) and (11) define the mathematical characteristics of the decision variables. 
Table 2. Table of notations.

\begin{tabular}{|c|c|}
\hline Symbol & Definition \\
\hline \multicolumn{2}{|l|}{ Sets: } \\
\hline$T$ & Set of time periods (indexed by $t$ and $p$ ) \\
\hline$L$ & Set of lines (indexed by $l$ and $k$ ) \\
\hline$S$ & Set of stops (indexed by $i$ ) \\
\hline$S^{l}$ & Set of stops on line $l\left(S^{l} \subset S\right)$ \\
\hline$C^{l k}$ & Set of transfer points on lines $l$ and $k\left(C^{l k} \subset S\right)$ (indexed by $q$ ) \\
\hline$D_{t}$ & Set of departures in period $t$ (indexed by $m$ and $n$ ) \\
\hline$D_{t}^{l}$ & Set of departures on line $l$ in period $t\left(D^{l} \subset D\right)$ \\
\hline \multicolumn{2}{|c|}{ Parameters: } \\
\hline$U$ & Length of the time periods (usually 1 hour) \\
\hline$C T_{t}^{l}$ & Average round-trip time of line $l$ in period $t$ \\
\hline$H \min _{t}^{l}$ & Minimum headway on line $l$ in period $t$ \\
\hline$H \max _{t}^{l}$ & Maximum headway on line $l$ in period $t$ \\
\hline$D w_{i t}^{l}$ & Average dwell time at stop $i$ on line $l$ in period $t$ \\
\hline$\delta_{t p}^{l k}$ & $\begin{array}{l}\text { Maximum allowable time window for a synchronized arrival from line } l \\
\text { in period } t \text { and line } k \text { in period } p \text { at a transfer point }\end{array}$ \\
\hline$R_{i, i+1, t}^{l}$ & $\begin{array}{l}\text { Average travel time between two consecutive stops } i \text { and } \\
i+1 \text { on line } l \text { in period } t\end{array}$ \\
\hline \multicolumn{2}{|c|}{ Decision variables: } \\
\hline$x_{t}^{l}$ & The interval between the beginning of period $t$ and the first departure on line $l$ in that period \\
\hline$Y_{m n q t p}^{l k}$ & $\begin{array}{l}\text { A binary variable which yields the value } 1 \text { if the } m \text { th departure of period } t \text { on line } l \text { meets } \\
\text { the } n \text {th departure of period } p \text { on line } k \text { at transfer point } q \text { within a time window } \delta^{l k} \\
\text { otherwise it yields the value } 0\end{array}$ \\
\hline$F S^{l}$ & Average number of required fleet size on line $l$ \\
\hline$H_{t}^{l}$ & Planned headway on line $l$ \\
\hline$A_{n i t}^{l}$ & Arrival time of departure $n$ to stop $i$ on line $l$ in period $t$ \\
\hline$N D_{t}^{l}$ & The number of departures on line $l$ in period $t$ \\
\hline
\end{tabular}

Of note, to ensure the practicality of the problem, the two following constraints should also hold:

$$
\begin{aligned}
& U \geq\left(N D_{t}^{l}-1\right) \cdot H \min _{t}^{l} \quad \forall l \in L, t \in T, \\
& U \leq N D_{t}^{l} \cdot H \max _{t}^{l} \quad \forall l \in L, t \in T .
\end{aligned}
$$

According to Constraints (12) and (13), departures that are planned for a period do not violate the corresponding time interval. The presented mathematical formulation is the most comprehensive form which can be simplified for implementation purposes.

\section{Solution approaches}

The proposed comprehensive model is widely applicable to networks with different sizes; therefore, the implementation approach is discussed in two sections for both small and large networks.

\subsection{Scale of the problem}

Although binary and discrete variables complicate the problem, they are often used in mathematical modeling, yielding a mixed integer programming problem. As described in the previous section, the principal decision variables of the proposed model, i.e., simultaneity and headway variables, are binary and discrete variables, respectively. Moreover, the nonlinearity of mathematical equations and constraints is, in some cases, unavoidable. For example, Constraint (8) that defines the simultaneity phenomenon cannot be efficiently linearized without increasing the number of constraints and variables, hence a more complicated problem. Furthermore, dimensions of the problem play a significant role in complicating the problem and increasing the computation time. The complexity of the proposed mathematical model which consists of binary and discrete variables, nonlinear constraints, and several dimensions can be usually evaluated by the number of integer variables of the problem. Variable 
$y_{m n q t p}^{l k}$ stands for the simultaneous arrivals of the vehicle $m$ in period $t$ on line 1 and vehicle $n$ in period $p$ on line $k$ at transfer stop q. Accordingly, there is a binary variable for the combination of every two trips on every two lines which intersect at stop $q$. Given that $L$ is the total number of lines on a network which, at some points, intersect with other lines, $D$ is the average number of departures planned on each line in a given period, $C$ is the total number of transfer stops, and $T$ is the number of time periods; then, the number of integer variables $y_{m n q t p}^{l k}$ would be $T^{2} \times C \times L^{2} \times D^{2}$. In a more realistic approach, if $C$ is substituted by the average transfer stops along each of two lines, the number of variables would be equal to $T^{2} \times C \times L^{2} \times D^{2}$ which would be a large number in real networks. Moreover, the number of variables associated with fleet size, $F S^{l}$, assuming $H=\left(H \max _{t}^{l}-H \min _{t}^{l}\right)+1$ as the number of possible values for headways on each line in each period, would be equal to $T \times L \times H$. Consequently, the feasibility of efficiently implementing the proposed model on real-world transit networks with different sizes is quite controversial.

Furthermore, with respect to the computational complexity theory, the basic model developed by Ceder et al. [14] with the objective of maximizing the number of simultaneous arrivals is proved to be an NP-hard problem [48] whose complexity provides enough incentive for the authors to consider heuristic algorithms as a solution approach. To this end, the attempted problem in this paper is classified as in the NP-hard class of problems.

\subsection{Converting the problem to a single objective problem}

In order to solve the problem using the single-objective solution approaches proposed in this paper, it should be converted into a single-objective problem. To this end, the linear Weighted Sum Method (WSM) was employed. Eq. (14) shows the objective function of the problem converted by the linear WSM:

$$
\begin{gathered}
\max Z=\left(C_{1} \sum_{m \in D_{t}^{l}} \sum_{n \in D_{t}^{k}} \sum_{q \in C^{l k}} \sum_{l \in L} \sum_{\substack{k \in L \\
l \neq k}} \sum_{t \in T}\right. \\
\left.\sum_{p \in T} y_{m n q t p}^{l k}-C_{2} \sum_{l \in L} F S^{l}\right) .
\end{gathered}
$$

Eqs. (15)-(17) show the additional constraints incorporated to the model using the mentioned method:

$$
\begin{aligned}
& C_{1}+C_{2}=1, \\
& C_{1} \geq 0 \\
& C_{2} \geq 0 .
\end{aligned}
$$

In order to convert the bi-objective mathematical problem into a single-objective one, the relative importance of the objectives should be assigned to their corresponding function as their weights. The weight values are quite important and delicate since they directly affect the final solution of the problem. In this respect, further studies are required for determining the weight values. There are also some popular methods used in conjunction with this purpose including brainstorming and Delphi methods. However, the ultimate decision should be made by the members of the management team who are known as the most experienced decisionmakers in this field. In this study, considering the monetary value of each simultaneous arrival which saves passengers' time and the cost of each vehicle to ensure more simultaneous arrivals, the weights are assigned as $C_{1}=0.23, C_{2}=0.77$ only to provide an illustrative example of using methods designed for single-objective models.

Finally, in order to scale the objectives with the same unit and magnitude, they are normalized according to Eq. (18):

$$
\tilde{F}_{i}=\frac{F_{i}(x)-F_{i, \min }}{F_{i, \max }-F_{i, \min }}
$$

where $F_{i \text {,min }}$ and $F_{i \text {, max }}$ are the minimum and maximum values for the objective function $i$, respectively, and the normalized objective function $\tilde{F}_{i} \in[0,1]$, $i=1,2, \ldots, m$ has the same space design as before [49]. For more perceivable depiction, two simplifications are made as shown in Eqs. (19) and (20), and using Eq. (18), the normalized objective function is presented in Eq. (21).

$$
\sum_{m \in D_{t}^{l}} \sum_{n \in D_{t}^{k}} \sum_{q \in C^{l k}} \sum_{l \in L} \sum_{\substack{k \in L \\ l \neq k}} \sum_{t \in T} \sum_{p \in T} y_{m n q t p}^{l k}=S
$$

$$
\begin{aligned}
& \sum_{l \in L} F S^{l}=F S \\
& Z=C_{1}\left(\frac{S-S_{\min }}{S_{\max }-S_{\min }}\right)-C_{2}\left(\frac{F S-F S_{\min }}{F S_{\max }-F S_{\min }}\right) .
\end{aligned}
$$

The resulting Eq. (21) is used as the objective function of the model in cases of using the single-objective solution methods.

\subsection{Small cities}

In small cities with small bus networks and only a few lines, a simplified version of the proposed model can be employed for timetable setting; however, the model needs to be solved for each period of a day. Thus, the variables and parameters lose the index $t$, and the 


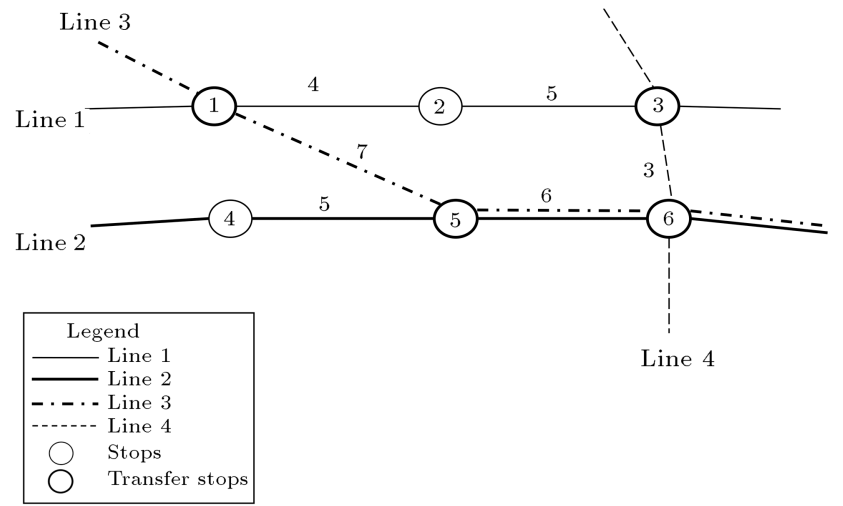

Figure 2. Example network.

simplified model could be efficiently solved using classic nonlinear mathematical methods.

In this section, a small numerical example is presented and solved using General Algebraic Modeling System (GAMS). Figure 2 illustrates a transit network with 4 lines and 6 stops. The planning horizon comprises 60 minutes (the morning peak hour) and the travel times between successive stops are mentioned in Figure 2. As illustrated in Figure 3, stops 1, 3, 5, and 6 are the transfer stops of at least two intersecting lines.

Table 3 presents the input data of the problem that includes the minimum and maximum allowable headways for each line, average dwell time of the vehicles at each stop, and average round-trip time on each line. Note that the dwell times of buses at the stops of the same line are assumed equal; hence, the parameter loses the stop index $i$.
Table 3. Required parameters (in minutes).

\begin{tabular}{ccccc}
\hline Line no. & $\boldsymbol{C T}^{\boldsymbol{l}}$ & $\boldsymbol{D w}^{\boldsymbol{l}}$ & $\boldsymbol{H}_{\min }^{\boldsymbol{l}}$ & $\boldsymbol{H}_{\max }^{\boldsymbol{l}}$ \\
\hline 1 & 33 & 3 & 2 & 7 \\
2 & 27 & 1 & 1 & 6 \\
3 & 51 & 5 & 3 & 10 \\
4 & 15 & 3 & 2 & 7 \\
\hline
\end{tabular}

The maximum allowable time window for the simultaneous arrival $\left(\delta^{l k}\right)$ at each transfer point in the network is set equal to two minutes. The proposed model is used to set timetables on the example network illustrated in Figure 2 using GAMS. The problem is coded in GAMS 23.0.2 and run using DICOPT solver which is based on the OA/ER/AP algorithm, the results of which are shown in Table 4 .

Table 4 demonstrates the scheduled departure times of vehicles on each line during the planning horizon. The last column shows the number of the required vehicles for each line. Figure 3 illustrates the occurrence of 11 of 23 consequent simultaneous arrivals at each transfer point at short intervals. Each line and its buses are recognized by a different geometric shape around the bus schematic and the time above is the arrival time of the bus at the transfer point. The same procedure can easily illustrate the remaining simultaneous arrivals.

As mentioned earlier, the best timetable would be the one that efficiently maintains the synchronization of different lines of a transit network requiring the minimum number of vehicles. The obtained results

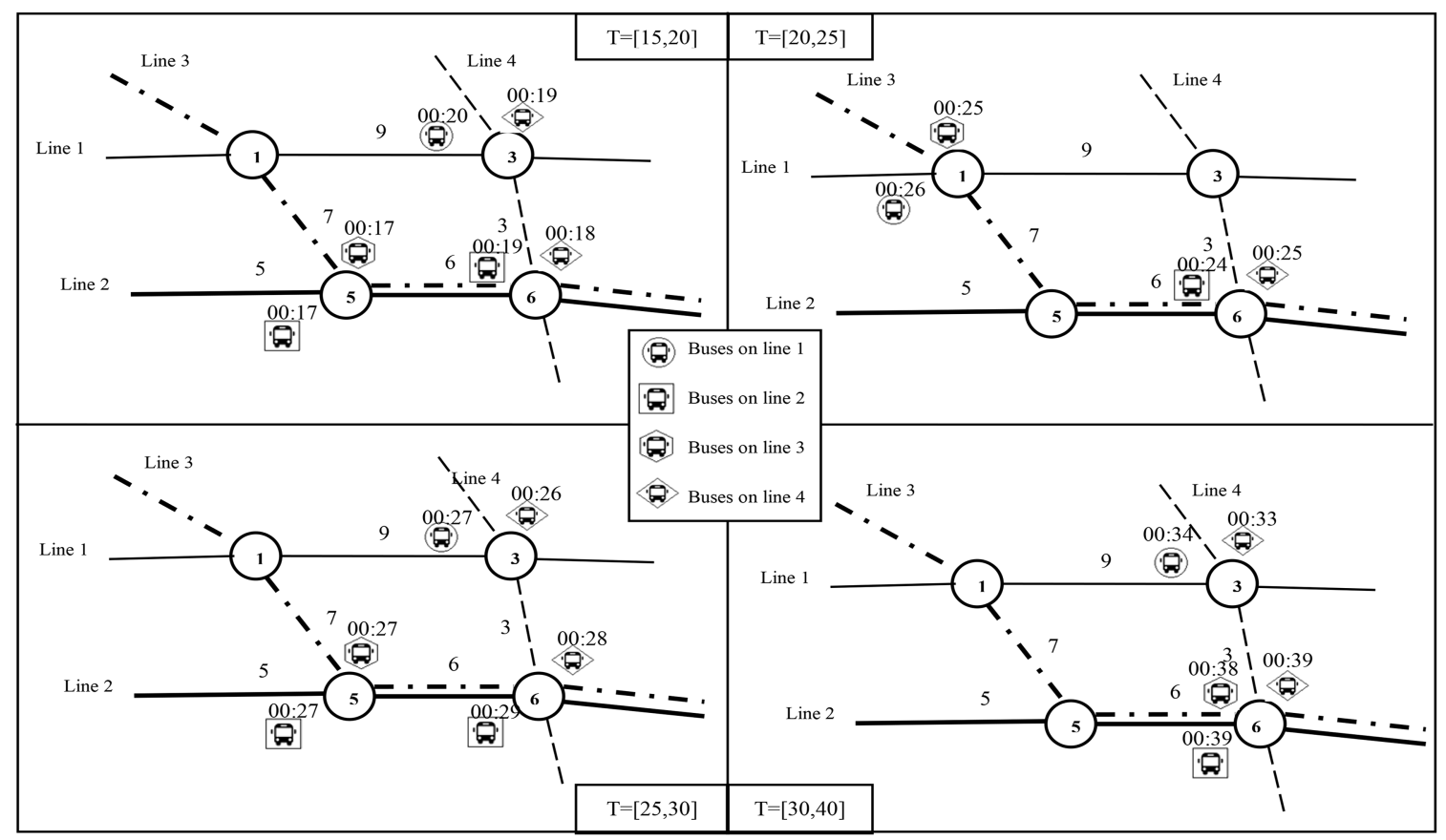

Figure 3. The first simultaneous arrivals at each transfer point. 
Table 4. Departure times of vehicles and the number of required vehicles for each line.

\begin{tabular}{cccccccccccccc}
\hline Line no. & $\mathbf{1}$ & $\mathbf{2}$ & $\mathbf{3}$ & $\mathbf{4}$ & $\mathbf{5}$ & $\mathbf{6}$ & $\mathbf{7}$ & $\mathbf{8}$ & $\mathbf{9}$ & $\mathbf{1 0}$ & $\mathbf{1 1}$ & $\mathbf{H}$ & $\mathbf{F S}$ \\
\hline $\mathbf{1}$ & 5 & 12 & 19 & 26 & 33 & 40 & 47 & 54 & - & - & - & 7 & 5 \\
$\mathbf{2}$ & 6 & 11 & 16 & 21 & 26 & 31 & 36 & 41 & 46 & 51 & 56 & 5 & 6 \\
$\mathbf{3}$ & 5 & 15 & 25 & 35 & 45 & 55 & - & - & - & - & - & 10 & 6 \\
$\mathbf{4}$ & 5 & 12 & 19 & 26 & 33 & 40 & 47 & 54 & - & - & - & 7 & 3 \\
\hline Total & & & & & & & & & & & & $\mathbf{2 0}$ \\
\hline
\end{tabular}

showed that the desired objectives could be achieved and the model would, by this definition, provide the optimal timetables under different conditions. In this regard, this model can be used for setting timetables on a small new network and improving the timetables of an existing one.

\subsection{Large cities}

Applying the proposed MINLP formulation to large transit networks using classic methods is quite difficult due to the nonlinear constraints, discrete search space, and the large size of the problem. Therefore, the metaheuristic algorithms were employed to efficiently solve the proposed model.

Among the most popular metaheuristic algorithms, GA have shown the highest efficiency in several cases in terms of transit scheduling problems [3947]. Therefore, both the proposed model and the GA were employed to set timetables on a real-world transit network with the minimum number of required vehicles. The process of using GA for solving the mentioned problem is described as follows.

\subsubsection{Metaheuristic approaches}

GA which is based on Darwin's theory of evolution is an optimization algorithm developed by Holland [50]. While considering the nature of the GA that can handle only problems with one objective function, the problem is converted into a single-objective problem using the linear weighted sum model and normalized, as shown in Eq. (21).

Solving the problem using the proposed mathematical model and GA requires several arrangements that are a part of the GA procedure, illustrated as a flowchart in Figure 4 (presented by Amiripour et al. [51]). As observed in the figure, first, an initial population of chromosomes is required. Finding a proper configuration for the chromosomes is an essential primary stage in implementation of GA. It not only affects the coding process but also provides a simple illustration of the problem for a better understanding. As shown in Figure 5, the configuration of the chromosomes is illustrated as a matrix.

Each row of this matrix contains the main variables of timetables for each transit line. The elements in each $1 \times 2$ block correspond to the time of the first departure and the headway of the corresponding period, respectively. The pattern is repeated for each line, thus forming the chromosome. According to Figure 5, after generating the initial population consisting of 200 chromosomes, each chromosome should be evaluated by a fitness function. In this case, similar to the small sample network problem, the fitness function that is the weighted linear combination of the introduced objective functions (Eqs. (2) and (3)) is normalized, as shown in Eq. (21). All of the constraints are taken into account in the process of generating the chromosomes, hence no need for penalty function. After the evaluation process, the algorithm starts an iterative loop that is repeated further for a specific number of times until no significant improvements are observed in the fitness value of the best chromosome.

Non-dominated Sorting Genetic Algorithm (NSGA-II) for multi-objective optimization, first proposed by Deb et al. [52] and Habibnejad-Ledari et al. [53], was proved to be an efficient solution approach. A similar procedure to what was described before was employed in the NSGA-II for a bi-objective problem; however, instead of a fitness value for each chromosome, there was a fitness vector consisting of different fitness values of various fitness functions.

The following section presents a real-world case as an illustration of the applicability of the proposed model and solution approaches in large-scale networks.

\subsubsection{A Case study: BRT network of Tehran}

In this section, the proposed model is applied to a large real-world network. Tehran BRT network, as a largescale network, consists of 9 lines (142 km), 8 of which have common stops between two or three lines, and according to the 2017 statistics, it serves an average of 2.25 million travelers on a daily basis with 900 buses. Figure 6 illustrates a plain scheme of the network. Red lines are the urban bus lines, and blue lines are the BRT lines. The proposed model and solution methods were employed to set coordinated timetables on this network.

Collecting the required data is the first step in solving the problem. Since the vehicles are equipped with Automatic Fare Collection (AFC) system and Automatic Vehicle Location (AVL) system, which are two main sources of data in a bus system, the required 


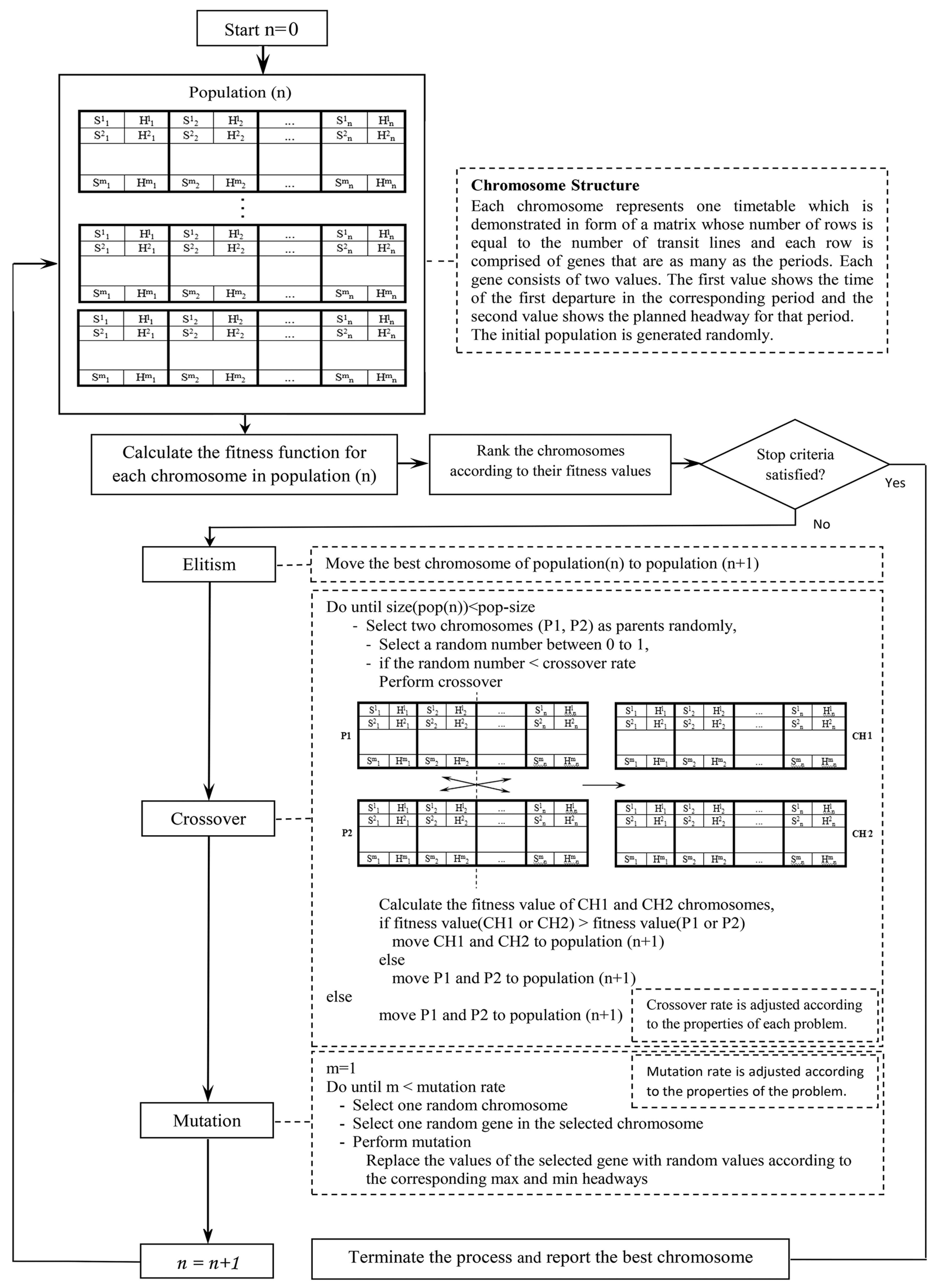

Figure 4. Genetic algorithm procedure flowchart [51]. 


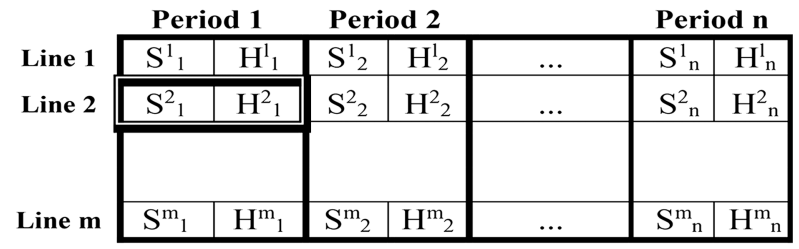

Figure 5. The configuration of the chromosomes.

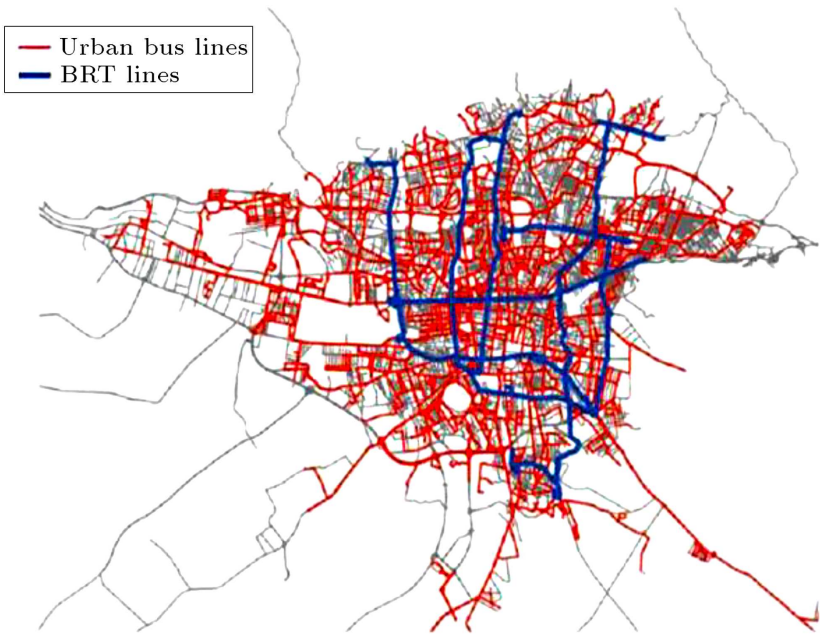

Figure 6. Tehran bus network.

data collection can be done conveniently. As an example, the AVL system records the time of opening and closing the bus doors at each stop. The required data includes the average travel time between two consecutive stops $\left(R_{i, i+1, t}^{l}\right)$, which can be calculated by subtracting the time at which the doors are opened at stop $i+1$ from the time at which the doors are last closed at stop $i$. Similarly, the average round-trip time of a line $\left(C T_{t}^{l}\right)$ can be calculated by subtracting the time at which the bus doors are closed at the last stop from the time at which the bus doors are opened at the first stop on the corresponding line. Moreover, the average dwelling time at each stop $\left(d w_{i t}^{l}\right)$ is the time between the opening and closing times of the bus doors at the same stop. For this purpose, the data for the first two weeks of January 2016 are collected, refined, and sorted according to the required input parameters of the model. The maximum allowable time window for a simultaneous arrival $\left(\delta_{t p}^{l k}\right)$ at each transfer point in the network is set equal to 1 minute. The planning horizon is divided into 17 hours and after tailoring the collected data in this format, the problem is coded in MATLAB 2017 using the GA. Then, the program is run on a PC with Pentium(R Dual-Core CPU E $53002.60 \mathrm{GHz}$ and 4.00 GB RAM; the results are given in Tables 5 and 6 .

Tables 4 and 5 demonstrate the obtained first departure times, headways, and the fleet size that are found by GA for each line for all periods during a day. Based on this information, a comprehensive timetable could be built that could minimize the waiting times for transferring passengers and synchronize the lines while decreasing the number of required vehicles. The

Table 5. The best first departure times found by Genetic Algorithm (GA) in each time period.

\begin{tabular}{|c|c|c|c|c|c|c|c|c|c|c|c|c|c|c|c|c|c|}
\hline & \multicolumn{17}{|c|}{ Time per. } \\
\hline Line no. & 1 & 2 & 3 & 4 & 5 & 6 & 7 & 8 & 9 & 10 & 11 & 12 & 13 & 14 & 15 & 16 & 17 \\
\hline 1 & 1 & 2 & 3 & 1 & 1 & 3 & 1 & 1 & 1 & 2 & 2 & 3 & 3 & 3 & 2 & 1 & 2 \\
\hline 2 & 1 & 3 & 1 & 4 & 2 & 3 & 6 & 1 & 1 & 4 & 3 & 1 & 1 & 4 & 4 & 6 & 2 \\
\hline 3 & 1 & 3 & 2 & 4 & 3 & 1 & 1 & 3 & 1 & 2 & 5 & 1 & 4 & 4 & 2 & 4 & 3 \\
\hline 4 & 3 & 4 & 5 & 2 & 2 & 3 & 6 & 2 & 6 & 2 & 3 & 3 & 3 & 2 & 3 & 4 & 5 \\
\hline 5 & 2 & 3 & 1 & 3 & 4 & 3 & 1 & 5 & 2 & 1 & 4 & 1 & 3 & 4 & 1 & 4 & 3 \\
\hline 7 & 2 & 1 & 3 & 4 & 6 & 7 & 2 & 5 & 3 & 5 & 2 & 1 & 7 & 3 & 6 & 3 & 7 \\
\hline 8 & 6 & 7 & 7 & 2 & 7 & 7 & 10 & 1 & 9 & 2 & 8 & 7 & 9 & 3 & 4 & 11 & 15 \\
\hline 10 & 5 & 1 & 5 & 2 & 6 & 1 & 6 & 3 & 1 & 3 & 6 & 2 & 2 & 1 & 6 & 4 & 2 \\
\hline
\end{tabular}

Table 6. The best headways and fleet sizes found by Genetic Algorithm (GA).

\begin{tabular}{ccccccccccccccccccc}
\hline & \multicolumn{11}{c}{ Time per. } \\
\hline Line no. & $\mathbf{1}$ & $\mathbf{2}$ & $\mathbf{3}$ & $\mathbf{4}$ & $\mathbf{5}$ & $\mathbf{6}$ & $\mathbf{7}$ & $\mathbf{8}$ & $\mathbf{9}$ & $\mathbf{1 0}$ & $\mathbf{1 1}$ & $\mathbf{1 2}$ & $\mathbf{1 3}$ & $\mathbf{1 4}$ & $\mathbf{1 5}$ & $\mathbf{1 6}$ & $\mathbf{1 7}$ & Fleet size \\
\hline $\mathbf{1}$ & 2 & 2 & 2 & 3 & 2 & 2 & 2 & 2 & 2 & 3 & 2 & 3 & 3 & 3 & 2 & 2 & 4 & 57 \\
$\mathbf{2}$ & 4 & 3 & 3 & 3 & 3 & 4 & 4 & 6 & 3 & 4 & 3 & 4 & 4 & 5 & 4 & 5 & 4 & 45 \\
$\mathbf{3}$ & 3 & 3 & 3 & 3 & 4 & 3 & 3 & 3 & 5 & 3 & 4 & 3 & 3 & 4 & 4 & 3 & 5 & 32 \\
$\mathbf{4}$ & 5 & 4 & 5 & 6 & 4 & 5 & 6 & 5 & 6 & 6 & 5 & 5 & 4 & 5 & 5 & 8 & 7 & 36 \\
$\mathbf{5}$ & 3 & 3 & 3 & 3 & 5 & 5 & 4 & 5 & 6 & 4 & 6 & 4 & 4 & 4 & 5 & 6 & 9 & 34 \\
$\mathbf{7}$ & 5 & 4 & 4 & 4 & 4 & 4 & 6 & 5 & 4 & 6 & 4 & 6 & 6 & 7 & 5 & 6 & 7 & 30 \\
$\mathbf{8}$ & 10 & 6 & 7 & 6 & 10 & 9 & 10 & 8 & 10 & 10 & 8 & 9 & 9 & 7 & 8 & 11 & 11 & 6 \\
$\mathbf{1 0}$ & 6 & 5 & 4 & 5 & 6 & 4 & 4 & 5 & 6 & 5 & 6 & 5 & 5 & 5 & 6 & 4 & 5 & 17 \\
\hline Sum & & & & & & & & & & & & & & & & & 25 \\
\hline
\end{tabular}


improvements and convergence trend of the GA are illustrated in Figure 7. As can be seen in the illustration, after about 8000 iterations, the algorithm converges to the presented solution with 10628 simultaneous arrivals and 257 vehicles. As explained before, NSGA-II is a powerful tool to obtain a more general solution to the bi-objective problem, especially in case the importance weights are unknown or a financial analysis of the possible solutions is necessary for the final decision to be made. In this case, five scenarios are considered for the current problem of Tehran BRT network $(\delta=$ $1, \ldots, 5$ minutes). For each scenario, all the Pareto optimal solutions are obtained and the Pareto fronts are illustrated in Figure 8. The parameter $\delta$ acts as a controlling parameter that directly affects simultaneous arrivals and its value should be selected according to the capacity of the system and the level of service. As $\delta$ decreases, simultaneity becomes difficult to occur. Therefore, $\delta=5$ is the least strict scenario that offers minimum level of service and $\delta=1$ is the strictest scenario that offers maximum service level. In case of the strict scenarios, the waiting time for the transferring passengers is reduced, but this improvement

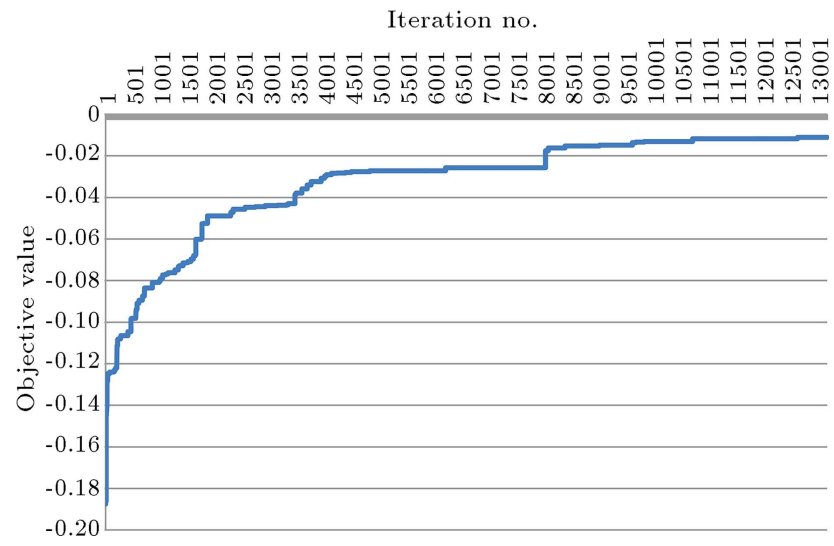

Figure 7. The GA convergence diagram.

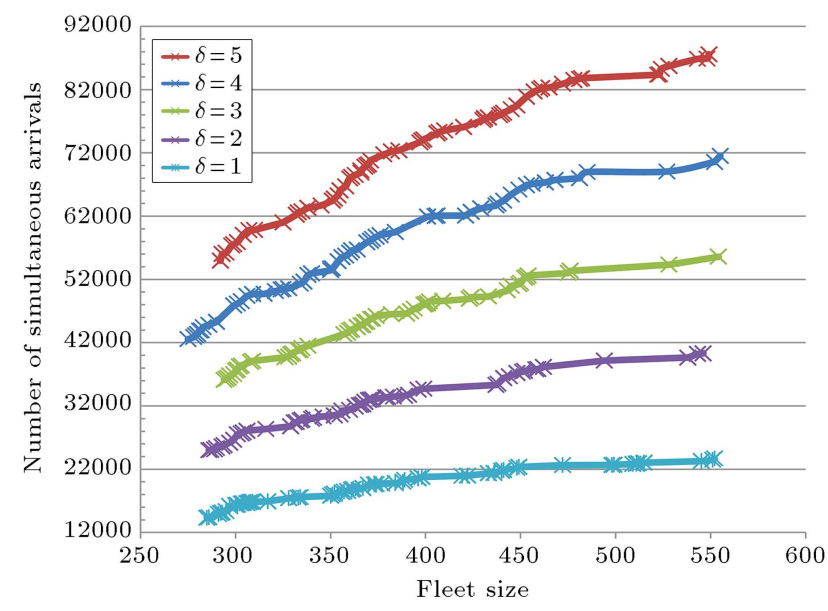

Figure 8. The Pareto optimal fronts of the Tehran Bus Rapid Transit (BRT) problem. would not be possible without a decrease in headways, demanding a higher number of vehicles and an increase in system costs. This finding proves that $\delta$ value is a sensitive, controversial, and impactful parameter. It is worth noting that as $\delta$ decreases, according to the increasing diagram gradient in Figure 8, a more significant increase in fleet size would be needed in order to obtain a higher degree of synchronization. A Pareto optimal front, as given Figure 8, can help decision-makers analyze the relationship between the objective values and weights closely and choose the best objective weight values according to the company's circumstances.

\section{Conclusions}

Scheduling (frequency determination and timetable setting) is one of the most important and complicated steps of the public transportation planning process. Synchronization is an important issue that should be considered during this step. It can have major effects on the attractiveness of public transit networks.

In this paper, by generalizing the primary concept of simultaneous arrivals and synchronization proposed by Ceder et al. [14], the frequency determination and timetable setting problem was formulated as a mixed-integer nonlinear programming model aimed at optimal synchronization of the timetables in a public transit network. The applicability of the proposed mathematical model was proved by its application to setting timetables on networks with different scales. In case of small networks, the problem was converted into a single-objective problem, the formulation was simplified, and the model was applied to a sample network with 4 lines and 6 stops as a case study. The problem was coded in General Algebraic Modeling System (GAMS) and a thoroughly reasonable timetable was obtained.

Since the classic methods are not capable of solving large-scale mathematical programming problems, two solution methods were proposed for these cases. First, the genetic algorithm was employed to use the single-objective problem in order to set timetables on the BRT network of Tehran consisting of 8 lines. Secondly, the NSGA-II was given to extract the Pareto optimal solutions and fronts in five different scenarios. The overall results showed that the proposed model could be easily used for synchronizing both small and large bus transit networks considering the vehicle costs by minimizing the required fleet size.

The problem discussed here is a highly complex problem that is under the influence of many correlative factors. It could be more realistic if the stochastic nature of some parameters such as average dwelling time at stops, average travel time between two consecutive stops, and average round-trip time 
of lines was incorporated into the model for future studies. Furthermore, such parameters as maximum headways and maximum allowable time window for synchronized arrivals that are associated with the level of service need thorough discussion by and decisions of the experts.

\section{References}

1. Liu, T., Ceder, A., Ma, J., and Guan, W. "Synchronizing public transport transfers by using intervehicle communication scheme: Case study", Transportation Research Record: Journal of the Transportation Research Board, 2417(1), pp. 78-91 (2014).

2. Ceder, A. "Public transit planning and operation: Theory, modeling and practice", In Elsevier, Butterworth-Heinemann, Oxford, UK (2007).

3. Desaulniers, G. and Hickman, M.D. "Public transit", Handbooks in Operations Research and Management Science, 14, pp. 69-127 (2007).

4. Guihaire, V. and Hao, J.K. "Transit network design and scheduling: A global review", Transportation Research Part A: Policy and Practice, 42(10), pp. 1251-1273 (2008).

5. Wu, Y., Yang, H., Tang, J., and Yu, Y. "Multiobjective re-synchronizing of bus timetable: Model, complexity and solution", Transportation Research Part C: Emerging Technologies, 67, pp. 149-168 (2016).

6. Ibarra-Rojas, O.J., Delgado, F., Giesen, R., and Muñoz, J.C. "Planning, operation, and control of bus transport systems: A literature review", Transportation Research Part B: Methodological, 77, pp. 38-75 (2015).

7. Wu, Y., Tang, J., Yu, Y., and Pan, Z. "A stochastic optimization model for transit network timetable design to mitigate the randomness of traveling time by adding slack time", Transportation Research Part C: Emerging Technologies, 52, pp. 15-31 (2015).

8. Ibarra-Rojas, O.J., López-Irarragorri, F., and RiosSolis, Y.A. "Multiperiod bus timetabling", Transportation Science, 50(3), pp. 805-822 (2015).

9. Nagatani, T. "Nonlinear-map model for bus schedule in capacity-controlled transportation", Applied Mathematical Modelling, 37(4), pp. 1823-1835 (2013).

10. Gershenson, C. and Pineda, L.A. "Why does public transport not arrive on time? The pervasiveness of equal headway instability", PloS One, 4(10), e7292 (2009).

11. Petersen, H.L., Larsen, A., Madsen, O.B., Petersen, B., and Ropke, S. "The simultaneous vehicle scheduling and passenger service problem", Transportation
Science, 47(4), pp. 603-616 (2012).

12. Kang, L. and Zhu, X. "A simulated annealing algorithm for first train transfer problem in urban railway networks", Applied Mathematical Modelling, 40(1), pp. 419-435 (2016).

13. Guo, X., Wu, J., Sun, H., Liu, R., and Gao, Z. "Timetable coordination of first trains in urban railway network: A case study of Beijing", Applied Mathematical Modelling, 40(17), pp. 8048-8066 (2016).

14. Ceder, A., Golany, B., and Tal, O. "Creating bus timetables with maximal synchronization", Transportation Research Part A: Policy and Practice, 35(10), pp. 913-928 (2001).

15. Eranki, A. "A model to create bus timetables to attain maximum synchronization considering waiting times at transfer stops", Master Thesis, University of South Florida (2004).

16. Currie, G. and Bromley, L. "Developing measures of public transport schedule coordination quality", 28th Australasian Transport Research Forum (2005).

17. Quak, C.B. "Bus line planning", Master Thesis, TU Delft (2003).

18. Ibarra-Rojas, O.J. and Rios-Solis, Y.A. "Integrating synchronization bus timetabling and single-depot single-type vehicle scheduling", ORP3 Meeting, Cádiz, Spain (2011).

19. Shafahi, Y. and Khani, A. "A practical model for transfer optimization in a transit network: Model formulations and solutions", Transportation Research Part A: Policy and Practice, 44(6), pp. 377-389 (2010).

20. Cevallos, F. and Zhao, F. "Minimizing transfer times in public transit network with genetic algorithm", Transportation Research Record: Journal of the Transportation Research Board, 1971(1), pp. 74-79 (2006).

21. Jansen, L.N., Pedersen, M.B., and Nielsen, O.A. "Minimizing passenger transfer times in public transport timetables", Proceedings of the Seventh Conference of the Hong Kong Society for Transportation Studies: Transportation in the Information Age, Hong Kong, China, pp. 229-239 (2002).

22. Chakroborty, P., Deb, K., and Subrahmanyam, P.S. "Optimal scheduling of urban transit systems using genetic algorithms", Journal of Transportation Engineering, 121(6), pp. 544-553 (1995).

23. Chakroborty, P., Deb, K., and Porwal, H. "A genetic algorithm based procedure for optimal transit systems scheduling", Proceedings of the Fifth International Conference on Computers in Urban Planning and Urban Management, Mumbai, India, pp. 330-0341 (1997).

24. Daduna, J.R. and VoB, S. "Practical experiences in schedule synchronization", In Computer-Aided Transit Scheduling, Lecture Notes in Economics and Mathe- 
matical Systems, Springer, Berlin, Heidelberg, 430, pp. 39-55 (1995).

25. Gao, Z., Sun, H., and Shan, L.L. "A continuous equilibrium network design model and algorithm for transit systems", Transportation Research Part B: Methodological, 38(3), pp. 235-250 (2004).

26. Bookbinder, J. and Désilets, A. "Transfer optimization in a transit network", Transportation Science, 26(2), pp. 106-118 (1992).

27. Ting, C.J. and Schonfeld, P. "Schedule coordination in a multiple hub transit network", Journal of Urban Planning and Development, 131(2), pp. 112-124 (2005).

28. Knoppers, P. and Muller, T. "Optimized transfer opportunities in public transport", Transportation Science, 29(1), pp. 101-105 (1995).

29. Yu, B., Yang, Z., Cheng, C., and Liu, C. "Optimizing bus transit network with parallel ant colony algorithm", Proceedings of the Eastern Asia Society for Transportation Studies, 5(1), pp. 374-389 (2005).

30. Poorzahedy, H. and Abulghasemi, F. "Application of ant system to network design problem", Transportation, 32(3), pp. 251-273 (2005).

31. Yang, Z.Z., Yu, B., and Cheng, C.T. "A parallel ant colony algorithm for bus network optimization", Computer-Aided Civil and Infrastructure Engineering, 22(1), pp. 44-55 (2007)

32. Peng, K. and Shen, Y. "A variable iterated greedy algorithm based on grey relational analysis for crew scheduling", Scientia Iranica, Transactions E, Industrial Engineering, 25(2), pp. 831-840 (2018).

33. Fleurent, C., Lessard, R., and Séguin, L. "August. transit timetable synchronization: Evaluation and optimization", Proceedings of the 9th International Conference on Computer-Aided Scheduling of Public Transport, San Diego, US, pp. 9-11 (2004).

34. Castelli, L., Pesenti, R., and Ukovich, W. "Scheduling multimodal transportation systems", European Journal of Operational Research, 155(3), pp. 603-615 (2004).

35. Rapp, M.H. and Gehner, C.D. "Transfer optimization in an interactive graphic system for transit planning", No. Intrm Rpt., pp. 27-33 (1967).

36. Deb, K. and Chakroborty, P. "Time scheduling of transit systems with transfer considerations using genetic algorithms", Evolutionary Computation, 6(1), pp.1-24 (1998).

37. Chakroborty, P., Deb, K., and Sharma, R.K. "Optimal fleet size distribution and scheduling of urban transit systems using genetic algorithms", Transportation Planning and Technology, 24(3), pp. 209-226 (2001).

38. Ngamchai, S. and Lovell, D.J. "Optimal time transfer in bus transit route network design using a genetic algorithm", Journal of Transportation Engineering, 129(5), pp. 510-521 (2003).

39. Pattnaik, S.B., Mohan, S., and Tom, V.M. "Urban bus transit route network design using genetic algorithm", Journal of Transportation Engineering, 124(4), pp. 368-375 (1998).

40. Chien, S., Yang, Z., and Hou, E. "Genetic algorithm approach for transit route planning and design", Journal of Transportation Engineering, 127(3), pp. 200207 (2001).

41. Bielli, M., Caramia, M., and Carotenuto, P. "Genetic algorithms in bus network optimization", Transportation Research Part C, 10(1), pp. 19-34 (2002).

42. Chakroborty, P. and Dwivedi, T. "Optimal route network design for transit systems using genetic algorithms", Engineering Optimization, 34(1), pp. 83-100 (2002).

43. Tom, V.M. and Mohan, S. "Transit route network design using frequency coded genetic algorithm", Journal of Transportation Engineering, 129(2), pp. 186-195 (2003).

44. Kuan, S.N., Ong, H.L., and Ng, K.M. "Solving the feeder bus network design problem by genetic algorithms and ant colony optimization", Advances in Engineering Software, 37(6), pp. 351-359 (2006).

45. Agrawal, J. and Mathew, T.V. "Transit route network design using parallel genetic algorithm", Journal of Computing in Civil Engineering, 18(3), pp. 248-256 (2004).

46. Fan, W. and Machemehl, R. "Optimal transit route network design problem with variable transit demand: genetic algorithm approach", Journal of Transportation Engineering, 132(1), pp. 40-51(2006).

47. Zhao, F. and Zeng, X. "Simulated annealing-genetic algorithm for transit network optimization", Journal of Computing in Civil Engineering, 20(1), pp. 57-68 (2006).

48. Ibarra-Rojas, O.J. and Rios, Y. "Synchronization of bus timetabling", Transportation Research Part B: Methodological, 46(5), pp. 599-614 (2012).

49. Abraham, A. and Jain, L. "Evolutionary multi objective optimization", In Evolutionary Multi Objective Optimization, Abraham, A., Jain, L., and Goldberg, R., Eds., pp. 1-6, Springer, London, UK (2005).

50. Holland, J.H., Adaptation in Natural and Artificial Systems: An Introductory Analysis with Applications to Biology, Control, and Artificial Intelligence, MIT press, Cambridge, MA (1992).

51. Amiripour, S.M., Ceder, A.A., and Mohaymany, A.S. "Designing large-scale bus network with seasonal variations of demand", Transportation Research Part C: Emerging Technologies, 48, pp. 322-338 (2014).

52. Deb, K., Pratap, A., Agarwal, S., and Meyarivan, T.A.M.T. "A fast and elitist multi objective genetic 
algorithm: NSGA-II", IEEE Transactions on Evolutionary Computation, 6(2), pp. 182-197 (2002).

53. Habibnejad-Ledari, H., Rabbani, M., and GhorbaniKutenaie, N. "Solving a multi-objective model toward home care staff planning considering cross-training and staff's preferences by NSGA-II and NRGA", Scientia Iranica, 26(5), pp. 2919-2935 (2019).

\section{Biographies}

Shervin Ataeian received her BSc in Industrial Engineering from University of Tabriz in and her MSc degree in the same field from Urmia University in 2014 and 2016, respectively. Her research interests include planning public transportation, modeling and scheduling bus network, and applications of operations research in transportation systems. She is currently expanding her studies in the field of transportation engineering.

Maghsud Solimanpur is a Professor of Industrial Engineering at the Department of Industrial Engineering, Urmia University, Urmia, Iran. He has received his BSc and MSc degrees from Sharif University of Technology in 1995 and 1997, respectively. He has been awarded a PhD degree from Indian Institute of Technology (IIT) Delhi in 2003. He has been awarded as the outstanding
PhD student by the Ministry of Science, Research and Technology of Iran in 2003. His research areas of interest include production and operations management, operations research, cellular manufacturing systems, facilities planning and layout, etc.

S.M. Mahdi Amiripour is an Assistant Professor at Toos Institute of Higher Education in Mashhad, Iran. His $\mathrm{PhD}$ is in Transportation Planning from Iran University of Science and Technology. His research interests include public transportation planning, designing and analysis of bus networks, bus networks modeling and scheduling, and public transport performance evaluation.

Ravi Shankar is a Professor of Operations and Supply Chain Management at the Department of Management Studies, Indian Institute of Technology (IIT) Delhi, India. Besides having a highly productive academic career at IIT Delhi, he has undertaken teaching in different highly prestigious international academic centers worldwide. He has supervised 50 defended/completed PhDs and over 100 masters-level theses for MTech and MBA degree programs. His research areas of interest include operations and supply chain management, decision sciences, business analytics, project management, sustainable freight transportation, etc. 